Review

\title{
"They've walked the walk": A systematic review of quantitative and qualitative evidence for parent-to-parent support for parents of babies in neonatal care
}

\author{
Harriet Hunt ${ }^{\mathrm{a}, *}$, Rebecca Abbott ${ }^{\mathrm{a}}$, Kate Boddy ${ }^{\mathrm{a}}$, Rebecca Whear ${ }^{\mathrm{a}}$, Leanna Wakely ${ }^{\mathrm{b}}$, Alison Bethel ${ }^{\mathrm{a}}$, \\ Christopher Morris ${ }^{\mathrm{a}}$, Susan Prosser ${ }^{\mathrm{c}}$, Andrew Collinson ${ }^{\mathrm{d}}$, Jennifer Kurinczuk ${ }^{\mathrm{e}}$, \\ Jo Thompson-Coon ${ }^{\mathrm{a}}$
}

${ }^{a}$ NIHR CLAHRC South West Peninsula (PenCLAHRC), University of Exeter Medical School, St Luke's Campus, Exeter, EX1 1TE, UK

${ }^{\mathrm{b}}$ Founder of SNUG (Supporting Neonatal Users and Graduates) and Volunteer at Royal Devon \& Exeter Hospital, Barrack Road, Exeter, EX2 5DW, UK

${ }^{\mathrm{c}}$ Lead Nurse for Children at the Royal Devon \& Exeter Hospital, Barrack Road, Exeter, EX2 5DW, UK

${ }^{\mathrm{d}}$ Consultant Paediatrician with Special Interest in Neonatology, Royal Cornwall Hospitals NHS Trust, Treliske, Truro, Cornwall, TR1 3LJ, UK

${ }^{\mathrm{e}}$ Director of the National Perinatal Epidemiology Unit, Nuffield Department of Population Health, University of Oxford, OX3 7LF, UK

\section{A R T I C L E I N F O}

\section{Keywords:}

Neonatal

Parent-to-parent support

Peer support

NNU

NICU

Preterm

Premature

Systematic review

\begin{abstract}
A B S T R A C T
The aim of this systematic review was to explore the effects and experiences of parent-to-parent support in neonatal intensive care from the perspectives of those giving, receiving, or implementing support. Electronic database searches (14 databases; February 2018) were supplemented with forward and backward citation chasing. Study selection, data extraction and quality appraisal were performed independently by two reviewers. Fourteen studies ( 6 quantitative and 8 qualitative) met our inclusion criteria. Four major themes were identified in the qualitative literature: 'trust', 'hope', 'information', and 'connecting'. Quantitative studies showed parentto-parent support increased perceptions of support, reduced maternal stress, and increased mothers' confidence in the ability to care for their baby. Whilst the rich qualitative evidence suggested mostly positive experiences of parent-to-parent support from all perspectives, robust trial evidence was lacking. Furthermore, differences in models for implementing parent-to-parent support provided limited opportunities to develop recommendations to guide best practice.

The protocol for this study was registered on PROSPERO, registration number CRD42018090569.
\end{abstract}

\section{Background}

Perinatal mental health problems carry a total economic and social long-term cost to society of about $£ 8.1$ billion for each one-year cohort of births in the UK (Bauer et al., 2014). Improving support for parents of premature babies is an important priority for parents, carers, health care professionals and relevant charities. Two priorities identified at the 2014 James Lind Alliance Pre-Term Birth Priority Setting Partnership workshop (Uhm et al., 2014) relate to improving support for parents: what should be included in packages of care to support parents and families/carers when a premature baby is discharged from hospital; and what emotional and practical support improves attachment and bonding and whether the provision of such support improve outcomes for premature babies and their families. The Picker Institute National Survey conducted in 2011 (Howell and Graham, 2011) also highlighted the need for improvements in parental support, suggesting there needs to be more clarity about how best to support the parent and how parents can best interact with staff in the neonatal intensive care unit (NICU). It has been suggested that parents may not feel welcome on the unit, may struggle to understand what is happening and find it difficult to watch others care for their baby (Guillaume et al., 2013).

Unsurprisingly there are higher levels of anxiety, depression and post-traumatic stress in mothers of pre-term babies than those whose children are born at term, and these challenges may persist long after discharge from the unit (Holditch-Davis et al., 2003; Phillips-Pula et al., 2013; Vigod et al., 2010; Miles et al., 2007). Combined with high levels of parental stress, these issues can affect the quality of the early parentchild relationship with long term implications for the health and wellbeing of both the parent and child (Guillaume et al., 2013). Reducing parent stress and improving self-esteem whilst on the unit and beyond

\footnotetext{
* Corresponding author.

E-mail address: h.a.hunt@exeter.ac.uk (H. Hunt).
} 
is likely to improve outcomes for parents and their children (Department of Health, 2009; Dennis, 2003; Hoey et al., 2008; Jolly et al., 2012; Repper and Carter, 2011).

A variety of interventions have been developed to support parents of preterm infants (Department of Health, 2009). Support forums or groups for parents are one such intervention, and can vary between being professionally led to peer (parent)-led. Peer support, defined as 'the provision of emotional, appraisal and informational assistance by a selected social network member who possesses experiential knowledge of a specific behaviour or stress and similar characteristics as the target population', is of particular interest (Dennis, 2003). Peer support has been shown to help people with a variety of health conditions in aspects such as depression, stress, Post-Traumatic Stress Disorder, emotional support and isolation (Hoey et al., 2008; Jolly et al., 2012; Repper and Carter, 2011; Charlesworth et al., 2008). Being able to talk to a peer (a parent) who is familiar with the experience of life in the neonatal unit may be uniquely beneficial. Indeed, studies of parent-to-parent support (P2P) have intimated that benefits include greater perceived empathy that parent peers are seen to have for the individuals they support, and opportunities for parent empowerment (Brett et al., 2011; Buarque et al., 2006). Whilst the evidence base for this in the NICU environment is less established, there is recognised growing awareness of the potential benefits (Hall et al., 2015).

The objective of this review therefore was to bring together studies exploring the experience and effects of P2P from the perspective of people giving and receiving support, and those involved in implementing P2P in the context of the provision of neonatal care.

\section{Methods}

This systematic review is reported in accordance with the Preferred Reporting Items for Systematic Reviews and Meta-Analyses (PRISMA) statement (Liberati et al., 2009). The protocol followed PRISMA-P reporting guidelines (Moher et al., 2015), was registered on the International Prospective Register of Systematic Reviews (PROSPERO), registration number CRD42018090569 and published in an open access journal (Hunt et al., 2018).

\subsection{Patient and public involvement}

The systematic review was conducted in collaboration with a studyspecific Parent Advisory Group (PAG). The group included parents with relevant and varied experience of having a baby in neonatal care and parents with experience of providing P2P. Four face to face meetings took place over the course of the project; between meetings the group was involved via a closed Facebook group. The PAG contributed to development of the protocol, commented on papers selected for inclusion, critiqued the synthesis, helped to interpret findings and coproduced a number of research outputs, including a plain language protocol summary (available on request).

\subsection{Eligibility criteria}

We were interested in any quantitative or qualitative study meeting the criteria below:

Population: Studies of parents of babies cared for in neonatal units accessing P2P at any time (during their time in hospital or back home in the community).

Interventions: P2P had to be led and provided by a parent (volunteer or paid) with experience of having an infant in neonatal care. We excluded studies reporting the effects or experiences of interventions provided by professionals, interventions which offered instruction or training to parents rather than support, studies of parent support specifically for families affected by bereavement or for those whose babies were receiving palliative care and studies which did not adequately describe the intervention.
Outcomes: Any experiences or effects of P2P from people offering, receiving or implementing $\mathrm{P} 2 \mathrm{P}$. We were also interested in any adverse experiences or effects of $\mathrm{P} 2 \mathrm{P}$.

We did not exclude by study design. Editorials, opinion pieces and letters were excluded.

\subsection{Search strategy}

We searched 14 bibliographic databases: Medline, Embase, PsycINFO, Social Policy and Practice (SPP), HMIC (via OvidSP), CINAHL Complete (via EBSCOhost), BNI, PQDT, ASSIA (via ProQuest), Social Sciences Citation Index, Conference Proceedings Citation Index Science and Social Sciences and Humanities (Web of Science, Clarivate Analytics) and the Cochrane Library (CDSR and CENTRAL) in February 2018 from inception. The search consisted of both free text and controlled vocabulary (e.g. MeSH) terms. The Medline search strategy is shown in Supplementary File 1. We carried out forwards and backwards citation chasing of the included papers using both SCOPUS and Web of Science. The search metrics are shown in Supplementary File 2. We also searched 12 websites and Nexis News in June 2018; details are listed in Supplementary File 3. In December 2018 we carried out a further database search in Medline, PsycINFO and CINAHL limited to the names of the specific UK programmes identified.

\subsection{Data collection and analysis}

Abstracts and titles of references retrieved by the search were each screened independently by two reviewers using the pre-specified inclusion/exclusion criteria. The full texts of potentially relevant studies were obtained and were also independently assessed for inclusion by two reviewers. Discrepancies were resolved by discussion; reference to a third reviewer was planned but not necessary.

\subsection{Data collection process}

Quantitative data were extracted by one reviewer and checked by another reviewer. As before, reference to a third reviewer was planned but not necessary. We used the template for intervention description and replication (TIDieR) checklist (Hoffmann et al., 2014) to inform the systematic data collection. For qualitative studies, we extracted details of the study aim, the sample, and the type and nature of the intervention/programme. We also collected data on the theoretical approach, the methods used to collect the data and the analytic processes. This process was conducted by two reviewers independently and discrepancies resolved through discussion.

\subsection{Quality appraisal}

We used the Effective Public Health Practice Project (EPHPP) quality assessment for quantitative data studies (Thomas et al., 2004). This tool enables an assessment of selection bias, study design, use of blinding, the level of confounding, data collection methods and data analysis, providing an overall summary rating of weak, moderate or strong. For qualitative studies, we used the Wallace criteria to determine the quality of reporting and the appropriateness of the method used (Wallace et al., 2004). The assessed criteria include theoretical perspective, appropriateness of question, study design, context, sampling, data collection, analysis, reflexivity, appropriateness generalisability, and ethics.

\subsection{Quantitative synthesis}

We used methods of quantitative synthesis as outlined by the Cochrane Handbook (Higgins and Green, 2011). The included quantitative studies reported a range of outcomes, which we grouped according to broad categories. There were insufficient homogenous data 
across studies to allow for a formal meta-analysis for any outcome. We therefore tabulated the data and summarised the effectiveness results narratively, grouping outcome measures by their broad category (e.g. psychological) and then by the specific measure (e.g. depression).

\subsection{Qualitative synthesis}

All qualitative outcomes were in the form of quotes (1st order), themes and concepts identified by study authors (2nd order), and themes and concepts identified by review authors (3rd order). The articles and the extracted data were read and re-read and the findings organized into sub-themes through discussion. Sub-themes were then grouped into main themes. The PAG took part in a reference group meeting to discuss the qualitative synthesis and establish relevance and transferability to a wider audience. We also held an impact conference with parents, neonatal staff and other stakeholders to explore these findings and implications for practice.

\subsection{Overarching synthesis}

We created an overarching synthesis to link the quantitative and qualitative evidence. We used the narrative synthesis methods outlined by Rodgers and colleagues (Rodgers et al., 2009), taking the five broad categories found in the quantitative synthesis and the sub-themes from the qualitative synthesis and looked at how they mapped across to each other, and whether the direction of effects and experiences were similar or not. We explored whether the qualitative findings could help explain the effects (or lack of) on quantitative outcomes.

\section{Results}

The electronic database searches found a total of 7431 articles. After title and abstract screening of 4593 articles, 118 full texts were retrieved for closer examination. Of these, 106 were excluded: the reasons for exclusion at the full text stage can be seen in the PRISMA flow diagram in Fig. 1. A total of 14 studies, reported in 15 articles, were included in the final review: 6 quantitative and 8 qualitative. The main study characteristics are shown in Table 1 .

\subsection{Study characteristics}

No studies from the UK were identified: 5 were from Canada, 8 from the USA and 1 from Finland (Table 1). One study focussed on neonatal staff (Rossman et al., 2012), one focussed on the parent support giver (Livermore, 1980), one focussed on all involved (parents, parent support giver and neonatal staff and managers) (Roman, 1988) and the remainder focussed on the parents (mothers) receiving support. Only one study included data from fathers, from the perspectives of both P2P givers and receivers (Roman, 1988). Four of 14 studies focussed on P2P specifically for breastfeeding (Rossman et al., 2011, 2012; Merewood et al., 2006; Niela-Vilen et al., 2016; Oza-Frank et al., 2014).

\subsection{Study quality}

The quality of quantitative studies is shown in Table 2. Selection bias, study design and accounting for confounders were well reported and mostly rated as moderate or strong. Blinding of intervention and outcome measurement was not rated as strong for any study, with four studies (Niela-Vilen et al., 2016; Oza-Frank et al., 2014; Minde et al., 1980; Preyde, 2007; Preyde and Ardal, 2003) rated as weak. Reporting on the data collection methods used and whether all participants were accounted for was variable. Overall study quality was mixed, with only one study rated strong (Roman et al., 1995), two studies as moderate (Merewood et al., 2006; Preyde, 2007; Preyde and Ardal, 2003) and three studies assessed as weak (Niela-Vilen et al., 2016; Oza-Frank et al., 2014; Minde et al., 1980).
The quality of the included qualitative studies was generally good, with the studies reported well and only a few concerns about bias across the studies (see Table 3). All but one (Macdonell et al., 2013) of the qualitative studies had clear research questions. All used appropriate study designs and described results that were clearly substantiated by the data. Most studies adequately described the setting and how data were collected, had adequate sample sizes and made reasonable claims about generalisability of findings. Less well reported were whether there were any theories/ideologies behind the research question and whether this influenced the study design. Only three studies reported reflexivity from the authors (Livermore, 1980; Roman, 1988; Morris, 2008).

\section{Quantitative synthesis}

Six studies (two randomised controlled trials, three case-control studies and one pre-post study) assessed the effect of P2P: three were specific to peer breast-feeding support (Merewood et al., 2006; NielaVilen et al., 2016; Oza-Frank et al., 2014). Data were not suitable for meta-analyses due to heterogeneity in study design and outcome measures. Outcomes were thus summarised narratively in the following areas: psychological, perceptions, interaction and behaviour, knowledge and understanding, and breastfeeding rates and attitudes. All results are shown as mean (SDs) where possible and reported as a comparison of the effect of P2P intervention compared to control.

\subsection{Psychological outcomes}

Two case-control studies assessed the effect of P2P on maternal anxiety and depression (Preyde and Ardal, 2003; Roman et al., 1995). Anxiety was measured by the Profile of Mood States (POMS) questionnaire at discharge, 1, 4 and 12 months in the study by Roman and by both State Anxiety Inventory and Trait Inventory at 4 months in the study by Preyde et al. (Preyde and Ardal, 2003). No difference in anxiety between those who had P2P and those who did not was found at discharge (Roman et al., 1995). Less anxiety in those who had received P2P however was found at 1 month [8.55 vs $15.84, \mathrm{p}<0.001$ ] (Roman et al., 1995) and 4 month follow ups ([6.57 vs 10.17, p > 0.05 (Roman et al., 1995)], [31.4 vs 38.6, $\mathrm{p}<0.05$ (Preyde and Ardal, 2003)) though at 12 months, Roman reported no observed difference between the two groups.

Depression, assessed using the Beck Depression Inventory (Preyde and Ardal, 2003) and as a sub-scale of the POMS (Roman et al., 1995) was reported as no different at discharge, 1,4 or 12 month in the study by Roman, but lower for those who received P2P at 4 months in the study by Preyde et al. [2.20 vs $4.88, \mathrm{p}<0.01]$. Maternal stress, assessed at 1 month using the Parental Stressor Scale (PSS), was reported to be significantly lower for those who had P2P compared to those who did not, across all 4 domains (infant appearance, parental role, sights and sounds, staff relations), with the total score 1.54 vs 2.93, $\mathrm{p}<0.001$ (Preyde and Ardal, 2003). There were significant differences in effects on self-esteem from discharge to 4 months, assessed using the Rosenberg scale: increasing in mothers who had received P2P and decreasing for those who had not, with 4 month values reported as being significantly higher for mothers in the P2P group (raw data not shown, $\mathrm{p}<0.05$ ) (Roman et al., 1995). No beneficial effects of P2P were reported for anger, confusion or fatigue at discharge, 1 or 4 months (Roman et al., 1995).

\subsection{Perceptions relating to care and support}

Three papers (Minde et al., 1980; Preyde, 2007; Preyde and Ardal, 2003) reporting on two case control studies, assessed outcomes relating to perceptions of either the ability to care or perceptions of care and/or support received. At the time of discharge, mothers who had received P2P reported higher satisfaction with nursing care $(4.3(0.7)$ vs $3.9(0.7)$, 

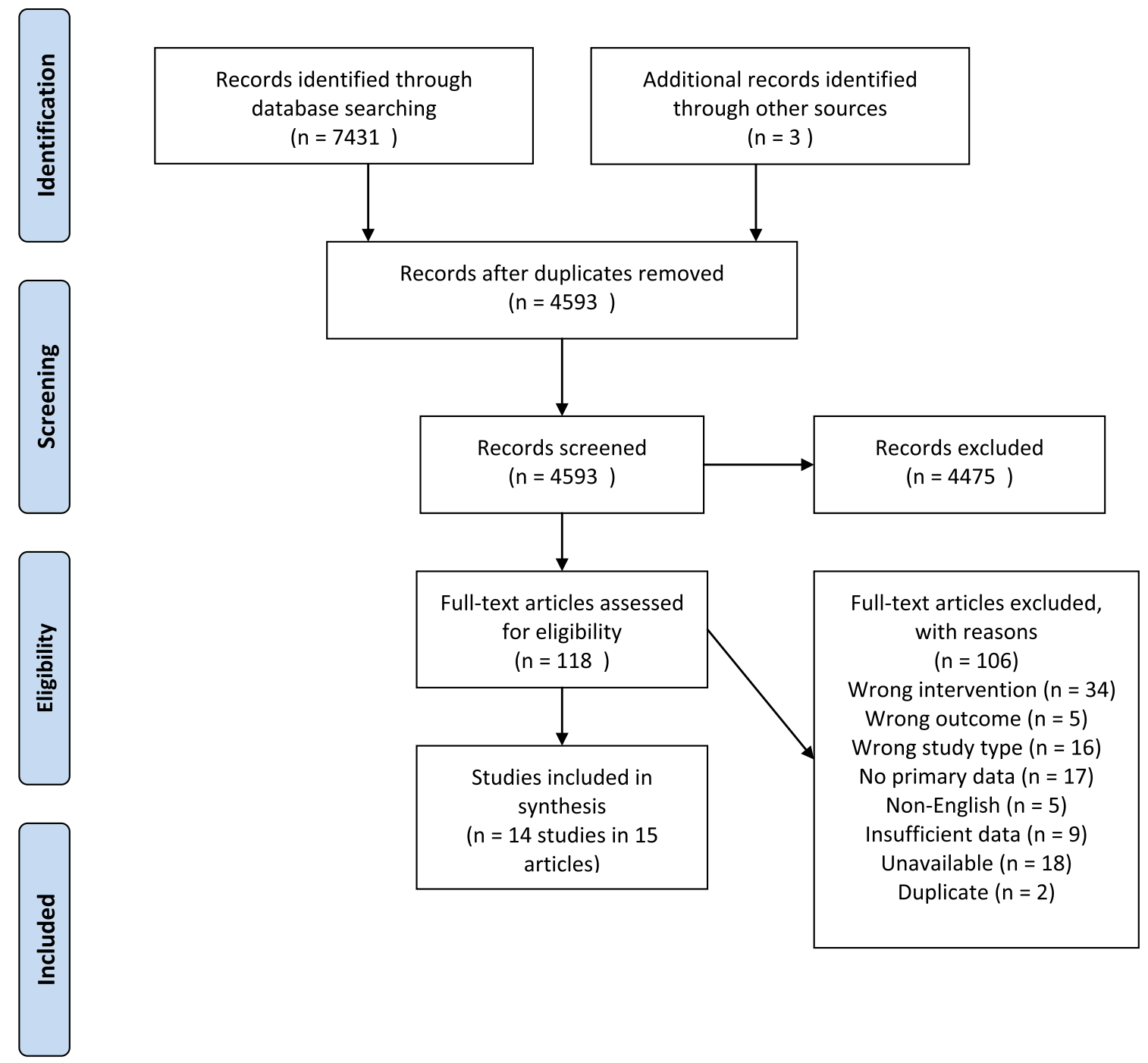

Fig. 1. PRISMA flow diagram of included studies.

$p<0.05)$ and medical care (4.6(0.6) vs 4.3(0.6), $p<0.02)$ whilst in the NICU and greater confidence in their ability to be able to care for their baby at home (3.8(1.2) vs 3.1(1.0), $p<0.05$ ) (Minde et al., 1980), compared to control groups. Greater perceived ability to care for their baby at 4 month follow up after discharge (raw data not shown, $t=2.49, p<0.05$ ), was also reported by mothers receiving P2P in the study by Preyde (2007). In this study, mothers who had received P2P also reported feeling more supported at 4 month follow up, as assessed by the Multidimensional Scale of Perceived Social Support [6.49 vs 5.48, $p<0.01$ ] (Preyde and Ardal, 2003), and more able to share their experience [raw data not shown, $U=121, p<0.01$ ] and have better listening support [raw data not shown, $U=192.5, p<0.05]$, than those who were not part of the P2P programme (Preyde, 2007).

\subsection{Interaction and parenting behaviours}

Two case control studies reported observations and self-reported items on mother-infant interaction and/or parental behaviours (Minde et al., 1980; Roman et al., 1995). Whilst in the NICU, mothers in the P2P group visited their infant more times per week than control mothers (4.5 visits (2.5) vs 3.1(1.9), $P=0.05$ ), and were also observed to touch, talk and 'look en-face' with their infant more than control mothers (data depicted graphically; $\mathrm{p}<0.05$ ), though smiling and simple looking were similar between the two groups (Minde et al., 1980). P2P mothers in this study also reported, at discharge, interacting more with other parents [3.8 interactions $(1.3)$ vs $2.0(1.0), p<0.01]$ than mothers in the control group. Observations of parenting routines related to feeding and sleeping at 1,2 and 3 months post discharge showed mostly no difference between those who had received support and those who had not (Minde et al., 1980). At one year follow up in the study by Roman et al. (1995), there was no difference in observations of family functioning (Feetham Family Functioning Scale) between P2P and control mothers, but higher scores were observed for parent-infant interaction (NCATS scale) [58.4(6.45) vs 52.32(11.6), $p<0.05]$ and for the quality of the home environment (HOME scale) 58.40(6.45) vs $52.32(11.6)$ in the families who had received P2P.

\subsection{Knowledge and understanding}

Two case-control studies reported on knowledge and understanding assessed using bespoke scales (Minde et al., 1980; Preyde and Ardal, 2003). Minde et al. (1980) found that at discharge from the NICU, mothers who had received P2P reported better understanding of their infant's condition [3.8(1.1) vs 2.8(1.0), $p<0.01$ ], had felt they had received better information during their NICU stay $[4.4(0.8)$ vs $3.5(1.0), \mathrm{p}<0.01$ ] and had greater knowledge of community resources available to them compared to control mothers [3.9(1.1 vs 2.7(1.0), $p<0.001$ ]. At 4 months follow up, Preyde et al. (Preyde and Ardal, 2003) found that mothers who received P2P reported a better understanding of their infant's medical condition compared to control 


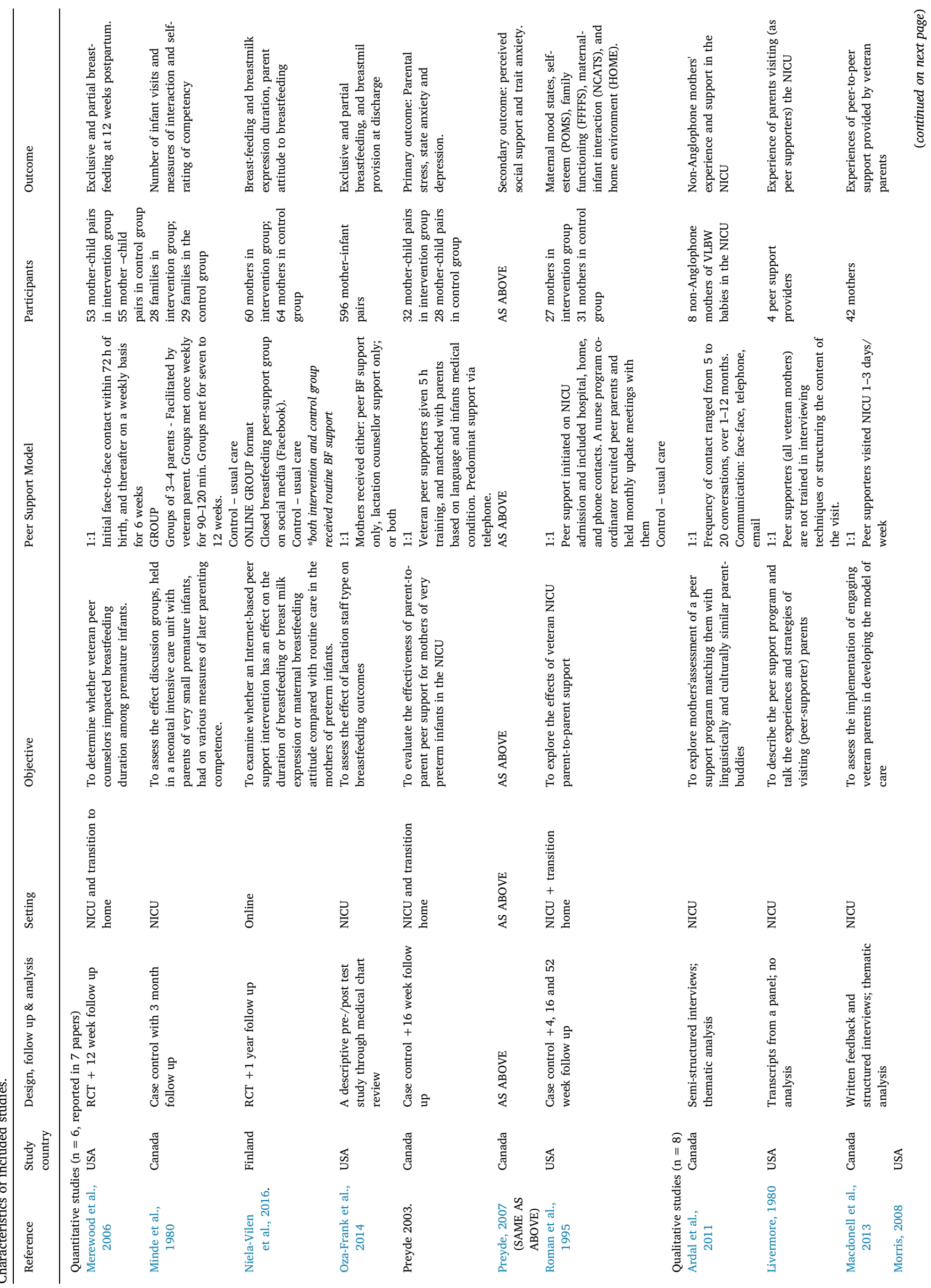


mothers [raw data not shown, $t=2.41, p<0.05]$.

\subsection{Breastfeeding rates and attitudes}

Two randomised controlled trials and one pre-post study assessed the effects of breast-feeding P2P on breastfeeding rates and duration (Merewood et al., 2006; Niela-Vilen et al., 2016; Oza-Frank et al., 2014). Both RCTs, one face to face (Merewood et al., 2006) and one internet-based (Niela-Vilen et al., 2016), found no evidence of effect of P2P on the number of mothers exclusively breastfeeding at 3-4 months post discharge. The proportion of babies who received any breast milk however was significantly higher in the face to face P2P trial, compared to control [OR $2.49(0.97-6.40), p=0.006]$, but there was no difference between groups in the internet-based P2P trial [HR 0.87 (0.53-1.4], $p=0.60$ ]. The pre-post study (Oza-Frank et al., 2014), which was partly retrospective, assessed the effect of lactation staff type on breastfeeding outcomes during the NICU stay and at discharge. Mothers who saw only P2P counsellors, or only lactation consultants, were less likely to provide exclusive or any breast milk during NICU stay or at discharge compared to mothers who saw both P2P counsellor and lactation consultant.

\section{Qualitative synthesis}

Eight studies described experiences of P2P. Data were grouped into 26 sub-themes or 'concepts', from which 4 main themes were identified. Concepts within the main themes are shown in Table 4.

\subsection{Theme one: trust}

Roman identified the role of those providing $\mathrm{P} 2 \mathrm{P}$ as friends who could share news and information however negative and whatever the topic, termed "bad news friends" (Roman, 1988). This was picked up by others, and talked about as 'keeping it real' or the ability of those providing P2P to be a 'reality check' (Roman, 1988; Macdonell et al., 2013; Morris, 2008; Ardal et al., 2011):

It's more realistic to hear that everything isn't always [rosy] but you will get through and every child is special no matter what they can or cannot do. [Mother of baby in NICU (Morris, 2008)]

The theme of those providing P2P as having "walked in the shoes" of the parents they supported was common (Rossman et al., 2011, 2012; Livermore, 1980; Roman, 1988; Macdonell et al., 2013; Ardal et al., 2011), acknowledging the value of shared experience to the parent being supported:

...there are only a few friends that have walked in your shoes so that they've made your experience of going through this a little easier to bear. [Mother of baby in NICU (Rossman et al., 2011)]

P2P providers recognised the value of timing (Livermore, 1980; Roman, 1988; Ardal et al., 2011). Experienced parent support was characterized by being 'timely' - that is, it occurred when the parent needed the support the most and was not limited by institutional roles or rules for appropriate contact. 'Judicious sharing' was labelled as the ability of peer supporters to share the right information at the right time with the parents they supported.

She waited for the precise moment to tell me what was next, so she was never ahead of the facts. [Mother of baby in NICU (Ardal et al., 2011)]

Understanding and a lack of critical judgment of the supported parent was identified as a key concept (Livermore, 1980; Roman, 1988; Morris, 2008; Ardal et al., 2011), with being a good listener a key requisite (Rossman et al., 2011, 2012; Livermore, 1980; Roman, 1988). Five studies (Livermore, 1980; Roman, 1988; Morris, 2008; Ardal et al., 2011) recognised the value of confiding and 'being able to vent' with a P2P provider: 
Table 2

Summary of quality appraisal for quantitative studies using the Effective Public Health Practice Project (EPHPP) tool.

\begin{tabular}{|c|c|c|c|c|c|c|c|}
\hline STUDY ID & Selection bias & Study design & Confounders & Blinding & Data Collection Methods & Withdrawals/Dropouts & OVERALL \\
\hline Merewood et al., 2006 & Moderate & Strong & Strong & Moderate & Poor & Moderate & MODERATE \\
\hline Minde et al., 1980 & Moderate & Moderate & Moderate & Weak & Weak & Weak & WEAK \\
\hline Niela-Vilen et al., 2016 & Strong & Strong & Strong & Weak & Moderate & Weak & WEAK \\
\hline Oza-Frank et al., 2014 & Moderate & Moderate & Moderate & Weak & Weak & $N A$ & WEAK \\
\hline Preyde 2003 \& Preyde, 2007 & Moderate & Moderate & Strong & Weak & Strong & Moderate & MODERATE \\
\hline Roman et al., 1995 & Strong & Moderate & Strong & Moderate & Strong & Moderate & STRONG \\
\hline
\end{tabular}

It is nice to be able to vent and [receive] feedback from other preemie parents. Before my daughter I had never known anyone who had a preemie. It was a hard thing to go through without people who understood my feelings. [Mother of baby in NICU (Morris, 2008)]

\subsection{Theme two: hope}

P2P providers were found to give parents confidence in their ability as a parent, and in their ability to parent within the NICU (Rossman et al., 2011, 2012; Livermore, 1980; Roman, 1988; Macdonell et al., 2013; Ardal et al., 2011):

She helped me to believe in myself, in my son, and to get the confidence that I needed. [Mother of baby in NICU (Ardal et al., 2011)]

This linked to a strong sense of reassurance that supported parents gained from the experienced parent providing P2P (Rossman et al., 2011, 2012; Livermore, 1980; Roman, 1988; Macdonell et al., 2013; Morris, 2008; Ardal et al., 2011):

When you see another parent who has been through what you are going through, it is reassuring to see them functioning. Like they were dressed, drove to the hospital, had makeup on. They talk about stuff matter of factly. [NICU parent (Roman, 1988)]

The concept of a 'changed perspective' for the supported parent was prominent in three studies (Livermore, 1980; Roman, 1988; Rossman et al., 2011) as was the place of the P2P supporter in normalising the role, environment and situation of the supported parent, and providing a role model for 'being a parent' (Livermore, 1980; Roman, 1988; Rossman et al., 2011; Morris, 2008; Ardal et al., 2011). 'Normal for NICU' was a concept explored in several studies (Livermore, 1980; Roman, 1988; Rossman et al., 2011; Macdonell et al., 2013; Ardal et al., 2011).

\subsection{Theme three: information and help}

The idea of 'NICU literacy' was explored across four studies (Livermore, 1980; Roman, 1988; Macdonell et al., 2013; Ardal et al., 2011) where P2P supporters were able to translate terms for new parents, identify different approaches and techniques and explain procedures that were unfamiliar - as well as point out environmental information such as where the milk room was.

They listen, they respond, they don't interrupt, don't get distracted, there is a silence that is not awkward, it is a waiting. Like they want to draw more, unless you experience it you can't understand it. [volunteer father (Roman, 1988)]

New parents were able to ask questions of peer parents that they felt uncomfortable asking NICU staff, and were able to discuss issues so that they knew the questions to ask the staff ((Roman, 1988; Morris, 2008):

Nurses know the answers, another mom can tell you what the questions are. Once we knew what to ask, the staff was great. We were "trained" really well. [NICU mother (Roman, 1988)]

Staff within the NICUs learnt from P2P supporters - this was highlighted in one study (Livermore, 1980). P2P supporters' role in encouraging new parents, in terms of feeling like parents, expressing hopes and fears, and in having physical contact with their babies, along with the P2P givers being a source of 'hands on' support, was identified in two studies (Livermore and Roman). Several studies (Livermore, 1980; Roman, 1988; Rossman et al., 2011) identified with the subtheme of support from P2P supporters being 'beyond the usual':

Although one visit and one or 2 phone calls is all that I usually do, I kept in touch with this mother for 8 or 9 months. We joke about it and say she bonded to me while she was in the hospital. The nice part of this story is that the baby was doing very well the last time I saw her. She was 9 months old and practically walking. [Volunteer mother (Livermore, 1980)]

\subsection{Theme four: connecting}

P2P helped reduce feelings of isolation in new NICU parents (Roman, 1988; Rossman et al., 2011; Macdonell et al., 2013; Morris, 2008):

I needed to not feel so isolated and I needed somewhere to vent my frustrations without being judged. It gave me a place to for lack of a better word "Share". Whether I was sharing pain or joy there was always someone there to listen. [New NICU parent (Morris, 2008)]

The role of supporting parents as extended family and friends was highlighted as important (Rossman et al., 2011; Macdonell et al., 2013; Morris, 2008):

The friends that we made in the NICU are also a huge support system for us. They understand what we have gone through and what we are still going through. [New mother (Morris, 2008)]

In one paper (Macdonell et al., 2013) the support offered and received was part of a two-way relationship in which the NICU staff benefitted as well as those being supported. Linked to this theme, one study (Livermore, 1980) highlighted the therapeutic benefit of giving support for P2P givers and the sense of feeling 'useful' to both the parents they supported and the wider organisation. This was not always a positive experience, and in the same study P2P givers reported anxiety and concerns that they were intruding on the new parent:

When I go into a hospital and get ready to knock on a new mother's door, there is always that twinge; am I intruding on this person, what if this person doesn't want any help, what if they don't want to hear what I've got to say .... They are not always really crazy to hear from us [peer supporter (Livermore, 1980)]

\section{Overarching synthesis}

There is overlap between the quantitative and qualitative evidence bases, but also some key differences. The quantitative research focusses on measuring self-reported outcomes from the mothers receiving support, with most attention being on perceptions of stress and anxiety and of confidence or self-efficacy in being able to care for their baby. Reductions in perceived stress and feelings of better support reported 


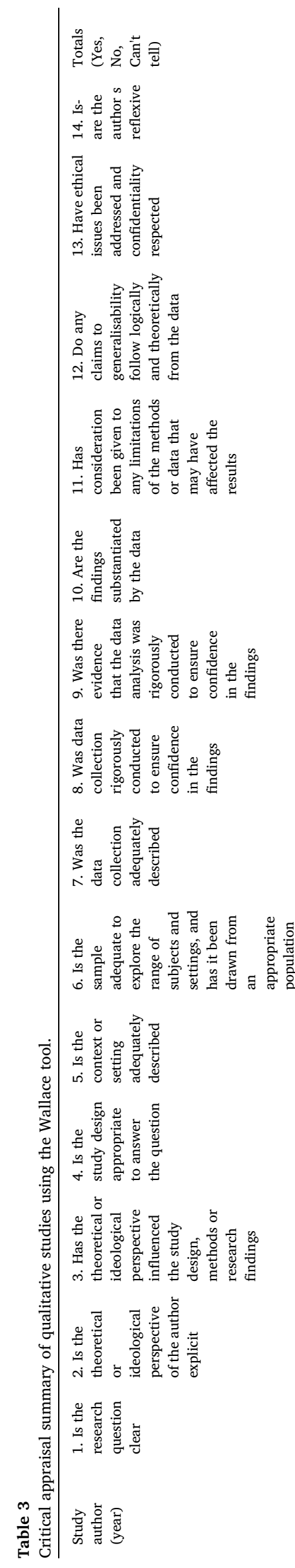

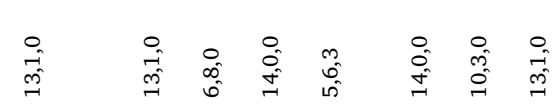

$z \quad z>>z>z z$

$\rtimes>z>z>\varnothing$

$\searrow>z>\varnothing \succ \succ$

$\searrow>\succ \succ \succ \succ \succ$

$\searrow \succ \succ \succ \succ \succ \succ$

$\succ>z>\quad \succ \succ \succ$

$\succ>z>\quad \succ z \succ$

$\rtimes>z>\quad \succ \succ \succ$

$\rtimes \succ \succ \succ \succ \succ$

$\searrow \succ \succ \succ \succ \succ$

$>\quad x>z>z>$

$\rtimes>z>z>z \varnothing$

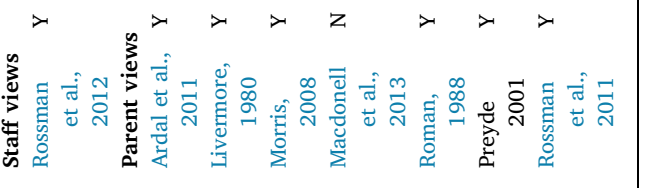


Table 4

Concepts developed from the main themes of qualitative evidence.

\begin{tabular}{ll}
\hline Theme & Concepts \\
\hline \multirow{2}{*}{ Trust } & Bad news friends \\
& Keeping it real \\
& 'Walking in shoes' \\
& Shared experiences \\
& Timing/'judicious sharing' \\
& Non-judgmental/understanding \\
& 'Being able to vent'/Confiding \\
& Listener \\
& Confidence \\
& Reassurance \\
& Changed perspective \\
& Normalising/Role Model/'Being a parent' \\
& Hands-on \\
"Normal for NICU" & Staff learnt from peers \\
Hope & 'NICU literacy' \\
& Being able to ask questions \\
& Having questions answered \\
Support beyond the usual \\
Right place, right time \\
Encouraging \\
Reduced isolation \\
Extended family + friends \\
Two way \\
Therapy \\
'Being useful' \\
\end{tabular}

by the mothers align well with the positive experiences of mothers feeling more hopeful, having someone who will listen and welcoming the shared understanding that came through strongly in the qualitative studies. While quantitative studies did explore effects on knowledge and understanding, the qualitative evidence synthesis expands this to provide rich detail about how mothers valued and trusted the experiential knowledge from the peer supporter, and in particular the ability of the peer supporter to use judicious timing in sharing information and experiences.

Impact on NICU staff and those providing P2P, explored in the qualitative research, was not measured in the quantitative research but are likely to be important considerations for implementation of P2P in NICUs. We identified no quantitative measures of how useful or helpful parents found P2P within the NICU, in transition or at home. We also found no quantitative measures of experiences of those providing $\mathrm{P} 2 \mathrm{P}$, in terms of either positive or negative effects. Very little evidence was identified on either qualitative or quantitative measures relating to P2P for fathers in the NICU or beyond. The only study to include data from fathers (Roman, 1988), found that father-to-father support did occur, when volunteer fathers were persistent in their attempts, and fathers themselves indicated that creative ways to involve fathers are needed because a mother support model will not work for fathers.

\section{Discussion}

This systematic review is the first, to our knowledge, to identify both quantitative and qualitative evidence of P2P interventions for families of babies cared for in neonatal units, and combine the findings in an integrated synthesis. Findings from observational and experimental studies, of mostly weak to moderate quality, suggest that P2P can result in less maternal anxiety and perceived stress post-discharge, and improvements in self-esteem up to the first 4 months post-discharge. Experimental evidence also suggests that mothers who received P2P reported better overall satisfaction with their care whilst in the NICU and feel more confident in being able to care for their child after discharge and in the first few months at home. Data from rich qualitative studies indicate that mothers feel that P2P from someone who has gone through a similar experience creates a shared understanding that helps to facilitate trust and the ability for confiding. Mothers receiving P2P experience a sense of hope for the future, and are helped to find a new normal. P2P also appears to afford connectedness and be regarded as a valued source of information. There were few studies of those giving or observing (implementing) P2P, but experiences were largely favourable and little negative experiences or adverse effects reported.

\subsection{Comparison to previous literature}

Previous research across a variety of health conditions and settings has shown that receiving support from someone who has experienced the same phenomena may help reduce feelings of isolation, anxiety, stress and depression (Hoey et al., 2008; Jolly et al., 2012; Repper and Carter, 2011; Charlesworth et al., 2008; Shilling et al., 2013). Our review suggests this extends to parents receiving P2P whilst in NICU and on discharge home, where maternal perceptions of stress and anxiety were reduced, and feelings of confidence or self-efficacy in being able to care for their baby were improved. Our findings are consistent with the suggestion that support from a peer affords psychological and physical health benefits through the sharing of social identity with others, and that this benefit also extends to the person offering support (Solomon, 2004). A previous mapping review of effective interventions for supporting, informing and communicating with parents who have had a preterm infant (Brett et al., 2011), suggested that provision of an environment where parents can meet and support each other was an effective way of supporting parents. Improvement in mothers who received P2P 'feeling supported' was one of the stronger findings in the quantitative evidence and this sense of being supported extended beyond discharge into the first few months of home. Communication, or knowledge exchange, has been suggested to be a key element of care for parents of babies cared for in neonatal units (Butt et al., 2013). 'Information and knowledge' and affording 'connections' were two of the main themes to come out of the qualitative findings, lending further weight to the benefit that P2P can have in the NICU.

\subsection{Strengths and limitations}

Our review followed best practice methods for systematic review and we did not restrict by study type or date (Hunt et al., 2018). Our stakeholder involvement throughout the review process helped to ensure the relevance and applicability to the context of current provision and our target audiences. Our strict definition of P2P, which excluded any involvement from health professionals as part of the support process, and/or the provision of education, did however mean that the number of studies we included was smaller than expected, and may therefore not fully reflect the true extent of P2P that may be available. We found a lack of detailed description and heterogeneity across definitions of P2P support, with models ranging from one to one in-person support between a veteran parent and a new parent, to group support, to online support through websites and closed chat groups. Furthermore, there was a large difference in models for implementing P2P support, some using a staff member as a co-ordinator, others employing a veteran parent to coordinate the support, and a range of training provision for peer supporters. These factors in combination meant we were unable to draw solid recommendations to guide best practice.

Whilst the rich qualitative evidence suggested mostly positive experiences of $\mathrm{P} 2 \mathrm{P}$ from the perspectives of those receiving it, robust trial evidence of effectiveness was lacking. There was also little research exploring the requirements needed to implement P2P. Whilst the need for comprehensive training and support for those providing $\mathrm{P} 2 \mathrm{P}$ is well recognised (Hall et al., 2015; Bourque et al., 2018; Fisher et al., 2018), there is as yet little evidence for how best to recruit and support those who will deliver P2P. We found no studies of P2P conducted in the UK, and no studies focussing on P2P for (or provided by) fathers exclusively. In fact the views and roles of fathers were underrepresented or missing entirely, with only one study including P2P for and by fathers as part of 
a more general P2P scheme. The importance of providing P2P for fathers has recently been highlighted by Fisher et al. in their review of the role of fathers in the neonatal unit (Fisher et al., 2018).

\subsection{Implications for practice}

The dearth of available evidence limits our ability to outline specific implications for practice. However, widespread discussion with parents, health care professionals and commissioners (from our stakeholder group) and informed by practical experience and the evidence within our systematic review, led to the development of a number of shared practice points:

- Local knowledge is invaluable - what works in some units may not work in others;

- P2P needs to take an individualised approach (everyone is different);

- $\mathrm{P} 2 \mathrm{P}$ is a positive addition for parents with babies being cared for in neonatal units;

- P2P provides an emotional support and can help reduce the experience of isolation;

- NICU staff can also learn from those providing P2P;

- P2P provides a valuable source of information and help within the NICU and beyond.

\subsection{Implications for research}

Our systematic review has identified a number of gaps in the evidence: i) studies conducted in the UK; ii) studies that assess the impact of P2P on fathers; iii) possible negative impacts of P2P (from the perspective of both those giving and those receiving); and iv) which models of P2P work for whom and in what circumstances. In addition to these gaps, there is also a clear need to develop knowledge to inform the successful implementation and sustainability of P2P.

\section{Conclusions}

Improving support for mothers and fathers of preterm infants remains a priority for neonatal service provision (Uhm et al., 2014; Howell and Graham, 2011). P2P appears to offer promise in this area, but before it can be routinely considered further research needs to robustly assess potential harms (for those providing and receiving care), and to tackle the pragmatic issue of how to implement and support P2P in practice.

\section{Ethical statement}

Ethical approval was not required.

\section{Conflicts of interest}

Leanna Wakely is the founder of SNUG and a neonatal parent-toparent peer supporter.

Rebecca Whear is a trustee of SNUG and a parent with lived experience of neonatal care.

Susan Prosser is a neonatal nurse by training and Lead Nurse for Children at the Royal Devon \& Exeter Hospital, Exeter, UK.

Andrew Collinson is a Consultant Paediatrician with Special Interest in Neonatology at Royal Cornwall Hospitals NHS Trust, Treliske, Truro, UK.

\section{Appendix A. Supplementary data}

Supplementary data to this article can be found online at https:// doi.org/10.1016/j.jnn.2019.03.011.

\section{Funding}

The work was funded by the National Institute for Health Research (NIHR) under its Research for Patient Benefit (RfPB) Programme (Grant Reference Number PB-PG-0416-20032).

\section{Role of the funding source}

This study presents independent research funded by the National Institute for Health Research (NIHR) Collaboration for Leadership in Applied Health Research and Care South West Peninsula (NIHR CLAHRC South West Peninsula). The views expressed are those of the author(s) and not necessarily those of the NHS, the NIHR or the Department of Health and Social Care.

\section{References}

Ardal, F., Sulman, J., Fuller-Thomson, E., 2011. Support like a walking stick: parentbuddy matching for language and culture in the NICU. Neonatal Netw. J. Neonatal Nurs. 30 (2), 89-98.

Bauer, Annette, Parsonage, Michael, Knapp, Martin, Iemmi, Valentina, Adelaja, Bayo, 2014. Costs of perinatal mental health problems [monograph]. London School of Economics and Political Science, London, UK.

Bourque, C.J., et al., 2018. Improving neonatal care with the help of veteran resource parents: an overview of current practices. In: Seminars in Fetal and Neonatal Medicine. Elsevier.

Brett, J., Staniszewska, S., Newburn, M., et al., 2011. A systematic mapping review of effective interventions for communicating with, supporting and providing information to parents of preterm infants. BMJ open 1, e000023. https://doi.org/10.1136/ bmjopen-2010-000023.

Buarque, V., et al., 2006. The influence of support groups on the family of risk newborns and on neonatal unit workers. J. Pediatr. 82 (4), 295-301.

Butt, M.L., et al., 2013. An integrative review of parent satisfaction with care provided in the neonatal intensive care unit. J. Obstet. Gynecol. Neonatal Nurs. 42 (1), 105-120.

Charlesworth, G., et al., 2008. Befriending carers of people with dementia: randomised controlled trial. BMJ 336 (7656), 1295-1297.

Dennis, C.-L., 2003. Peer support within a health care context: a concept analysis. Int. J. Nurs. Stud. 40 (3), 321-332.

Department of Health, 2009. Toolkit for High-Quality Neonatal Services.

Fisher, D., et al., 2018. Fathers in neonatal units: improving infant health by supporting the baby-father bond and mother-father coparenting. J. Neonatal Nurs. 24 (6), 306-312.

Guillaume, S., et al., 2013. Parents' expectations of staff in the early bonding process with their premature babies in the intensive care setting: a qualitative multicenter study with 60 parents. BMC Pediatr. 13 (1), 18.

Hall, S., et al., 2015. Recommendations for peer-to-peer support for NICU parents. J. Perinatol. 35 (S1), S9.

Higgins, J.P.T., Green, S. (Eds.), 2011. Cochrane Handbook for Systematic Reviews of Interventions, The Cochrane Collaboration: Available from: www.handbook. cochrane.org.

Hoey, L.M., et al., 2008. Systematic review of peer-support programs for people with cancer. Patient Educ. Counsel. 70 (3), 315-337.

Hoffmann, T.C., et al., 2014. Better reporting of interventions: template for intervention description and replication (TIDieR) checklist and guide. BMJ 348, g1687.

Holditch-Davis, D., et al., 2003. Posttraumatic stress symptoms in mothers of premature infants. J. Obstet. Gynecol. Neonatal Nurs. 32 (2), 161-171.

Howell, E., Graham, C., 2011. Patients' Experiences of Neonatal Care. A Report on the Findings of a National Survey. Picker Institute Europe.

Hunt, H., et al., 2018. Parent-to-parent support interventions for parents of babies cared for in a neonatal unit-protocol of a systematic review of qualitative and quantitative evidence. Syst. Rev. 7 (1), 179.

Jolly, K., et al., 2012. Systematic review of peer support for breastfeeding continuation: metaregression analysis of the effect of setting, intensity, and timing. BMJ 344 , d8287.

Liberati, A., et al., 2009. The PRISMA statement for reporting systematic reviews and meta-analyses of studies that evaluate health care interventions: explanation and elaboration. PLoS Med. 6 (7), e1000100.

Livermore, N., 1980. The visiting parent: a personal approach to helping parents of infants-at-risk. Infant Ment. Health J. 1 (3), 176-185.

Macdonell, K., et al., 2013. Implementing family-integrated care in the NICU: engaging veteran parents in program design and delivery. Adv. Neonatal Care 13 (4), 262-269.

Merewood, A., et al., 2006. The effect of peer counselors on breastfeeding rates in the neonatal intensive care unit: results of a randomized controlled trial. Arch. Pediatr. Adolesc. Med. 160 (7), 681-685.

Miles, M.S., et al., 2007. Depressive symptoms in mothers of prematurely born infants. J. Dev. Behav. Pediatr. 28 (1), 36-44.

Minde, K., et al., 1980. Self-help groups in a premature nursery-a controlled evaluation. J. Pediatr. 96 (5), 933-940.

Moher, D., et al., 2015. Preferred reporting items for systematic review and meta-analysis protocols (PRISMA-P) 2015 statement. Syst. Rev. 4 (1), 1. 
Morris, H., 2008. Premature Birth and Online Social Support: the Parents' Perspective. Texas Woman's University, pp. 196 p-196 pp.

Niela-Vilen, H., et al., 2016. Breastfeeding preterm infants - a randomized controlled trial of the effectiveness of an Internet-based peer-support group. J. Adv. Nurs. 72 (10), 2495-2507.

Oza-Frank, R., Bhatia, A., Smith, C., 2014. Impact of peer counselors on breastfeeding outcomes in a nondelivery NICU setting. Adv. Neonatal Care 14 (4), E1-E8.

Phillips-Pula, L., et al., 2013. Caring for a preterm infant at home: a mother's perspective. J. Perinat. Neonatal Nurs, 27 (4), 335-344.

Preyde, M., 2007. Mothers of very preterm infants:perspectives on their situation and a culturally sensitive intervention. Soc. Work. Health Care 44 (4), 65-83.

Preyde, M., Ardal, F., 2003. Effectiveness of a parent "buddy" program for mothers of very preterm infants in a neonatal intensive care unit. CMAJ Canadian Medical Association Journal 168 (8), 969-973.

Repper, J., Carter, T., 2011. A review of the literature on peer support in mental health services. J. Ment. Health 20 (4), 392-411.

Rodgers, M., et al., 2009. Testing methodological guidance on the conduct of narrative synthesis in systematic reviews: effectiveness of interventions to promote smoke alarm ownership and function. Evaluation 15 (1), 49-73.

Roman, L.A.W., 1988. Creating mothering for preterm infants: a grounded theory of veteran parent support initiated in a neonatal intensive care setting. In: Department of Child and Family Ecology. Michigan State University, pp. 249.

Roman, L.A., et al., 1995. Parent-to-parent support initiated in the neonatal intensive care unit. Res. Nurs. Health 18 (5), 385-394.
Rossman, B., et al., 2011. "They've walked in my shoes": mothers of very low birth weight infants and their experiences with breastfeeding peer counselors in the neonatal intensive care unit. J. Hum. Lactation 27 (1), 14-24.

Rossman, B., Engstrom, J.L., Meier, P.P., 2012. Healthcare providers' perceptions of breastfeeding peer counselors in the neonatal intensive care unit. Res. Nurs. Health 35 (5), 460-474.

Shilling, V., Morris, C., Thompson-Coon, J., Ukoumunne, O., Rogers, M., Logan, S, 2013. Peer support for parents of children with chronic disabling conditions: a systematic review of quantitative and qualitative studies. Developmental Medicine \& Child Neurology 55 (7), 602-609.

Solomon, P., 2004. Peer support/peer provided services underlying processes, benefits, and critical ingredients. Psychiatr. Rehabil. J. 27 (4), 392.

Thomas, B., et al., 2004. A process for systematically reviewing the literature: providing the research evidence for public health nursing interventions. Worldviews EvidenceBased Nurs. 1 (3), 176-184.

Uhm, S., Alderdice, F., Brady, I., et al., 2014. PS-052 Setting Preterm Birth Research Priorities With Multiple Professions And Service Users In The UK. Archives of Disease in Childhood 99, A130-A131.

Vigod, S.N., et al., 2010. Prevalence and risk factors for postpartum depression among women with preterm and low-birth-weight infants: a systematic review. BJOG An Int. J. Obstet. Gynaecol. 117 (5), 540-550.

Wallace, A., et al., 2004. Meeting the challenge: developing systematic reviewing in social policy. Pol. Polit. 32 (4), 455-470. 